\title{
Gamma Knife for Cushing disease - time for a reappraisal?
}

\section{Frederic Castinetti and Thierry Brue}

The first large-scale, multicentric analysis of long-term results with Gamma Knife (Elekta) radiosurgery in the therapeutically challenging area of Cushing disease shows that, in selected patients, this radiation technique enables hormonal control to be achieved in most patients with relatively few adverse effects but with a significant risk of disease recurrence.

Refers to Mehta, G. U. et al. Stereotactic radiosurgery for Cushing's disease: results of an international, multicenter study. J. Clin. Endocrinol. Metab. http://dx.doi.org/10.1210/jc.2017-01385 (2017)

The treatment of Cushing disease remains one of the most difficult tasks for endocrinologists dealing with pituitary diseases, mainly because corticotroph pituitary adenomas do not behave like other secreting pituitary tumours. Long-term recurrence of Cushing disease after trans-sphenoidal surgery is close to $20 \%$ (even in patients with the best prognostic factors), much higher than the recurrence rate in patients with acromegaly or prolactinomas ${ }^{1}$. Despite the fact that trans-sphenoidal surgery is still the first-line treatment for Cushing disease, further studies are needed to determine the most effective therapeutic options when the disease reoccurs. Medical treatments aimed at normalizing cortisol levels all have the same limitation; namely, that they cannot cure the patients. These treatments, each with their own specific adverse effects and costs, are rarely maintained long term in patients who are diagnosed, on average, in the fourth decade of life ${ }^{2}$. Bilateral adrenalectomy is obviously a curative option for hypercortisolism and has a minimal risk of endocrine recurrence ${ }^{3}$; however, the primum non nocere precept should be kept in mind as bilateral adrenalectomy per se will replace one disease with another harmful one (that is, adrenal insufficiency) ${ }^{4}$. Over the past 25 years, radiation techniques have improved through better accuracy in target definition, enabling increased efficacy with fewer adverse effects. However, use of these techniques remains a matter of controversy precisely because of the fear of long-term adverse effects. to obtain maximal efficacy should challenge commonly accepted ideas (which are based on conventional radiotherapy) regarding the prolonged period of time needed to obtain maximal efficacy with radiation techniques. Of note, recurrences were observed in almost $20 \%$ of patients after GKS. This finding shows that, in contrast to conventional radiotherapy, definitive control of hypercortisolism is not guaranteed when remission is obtained with GKS; long-term follow-up is mandatory to screen for recurrences (median time to recurrence in the study by Mehta et al. was $38 \pm 44$ months). The recurrence rate might also have slightly biased the overall efficacy of the procedure, as the mean follow-up was 5 years and some patients presented with disease recurrence after a longer period of time.

Another point of importance is radiation-induced adverse effects. Mehta and colleagues observed new endocrine deficits (hypopituitarism) and cranial neuropathy in their cohort (at rates of $25 \%$ and $3 \%$, respectively). The rate of induced pituitary deficiency is in keeping with previous studies; the risk increases with time after the procedure and a cautious follow-up should thus be maintained on a long-term basis. By contrast, the rate of cranial neuropathy was greater than expected. Of note, and as mentioned by the authors, 16 patients had received previous radiotherapy, which was shown to be a ever, as with other studies, the low number of patients compromised generalizability of the results. It is precisely this limitation that makes the recently published study by Mehta and colleagues of particular interest ${ }^{6}$. Their study is indeed the first multicentre, international study aimed at defining the efficacy and endocrine adverse effects of GKS in patients with Cushing disease.

Briefly, of 278 patients, cumulative control of hypercortisolism was obtained in $80 \%$ at the 10-year follow-up. The results should, however, be tempered by the fact that in $11 \%$ of these patients symptoms were controlled by medical treatments at the last follow-up (although they were not controlled by the same medical treatment before GKS), and a recurrence rate of $18 \%$ was observed on a long-term basis. GKS thus enabled cure in $50 \%$ of the 278 patients treated after a mean follow-up of 5 years. The time to achieve remission was shorter than that usually expected (14.5 months). This fairly short time 
statistically significant predictive factor for cranial damage. The high number of patients previously treated with radiotherapy might also have biased the GKS efficacy results, as it is well established that conventional radia-tion therapy needs years to be fully effective and a concomitant efficacy of both GKS and radiotherapy cannot be excluded. In such a large study, a more thorough analysis of long-term, non-endocrine adverse effects would have been of interest. In particular, although endocrine adverse effects are well known, endocrinologists are mainly concerned about the risks of non-endocrine adverse effects such as strokes, brain tumours or cognitive dysfunc-tion, as have been reported with conventional radiotherapy ${ }^{7}$. This point was not evaluated by Mehta and co-workers. Although the prin-ciples of GKS should theoretically limit these types of adverse effect, long-term data are still lacking. Future studies aimed at differentiating the sequelae of long-term exposure to a hyper-secreting state (particularly for acromegaly or Cushing disease) versus the adverse effects of GKS are clearly needed to reassure both clinicians and patients.

To conclude, as recently shown in the French Acromegaly Registry, the proportion of patients treated by radiation techniques has drastically decreased over the past 20 years ${ }^{8}$. This decrease is in part due to more effective antisecretory drugs and in part to incorrectly attributing the adverse effects reported with older radiation techniques to newer radiation techniques. The large international multicentre study by Mehta et al. clearly shows that therapeutic algorithms on Cushing disease should leave ample room for GKS and other new radiation techniques after unsuccessful surgery, albeit with the caveat that not all patients can benefit from these treatments. A clearly defined target on MRI is mandatory to obtain good efficacy while preserving pituitary function, a condition that is not frequently met as most patients are operated on for a microadenoma and usually have a negative pituitary MRI at the time of recurrence. In selected patients, GKS thus seems a safe and effective technique that should be systematically considered, provided long-term results confirm the reassuring safety data on non-endocrine parameters.

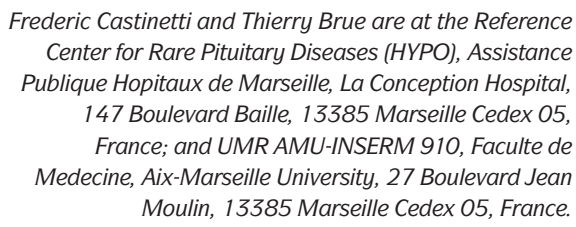

Frederic Castinetti and Thierry Brue are at the Reference Center for Rare Pituitary Diseases (HYPO), Assistance Publique Hopitaux de Marseille, La Conception Hospital, 147 Boulevard Baille, 13385 Marseille Cedex 05, France; and UMR AMU-INSERM 910, Faculte de Medecine, Aix-Marseille University, 27 Boulevard Jean Moulin, 13385 Marseille Cedex 05, France.

thierry.brue@ap-hm.fr; frederic.castinetti@p-hm.fr

1. Patil, C. G. et al. Late recurrences of Cushing's disease after initial successful transsphenoida surgery. J. Clin. Endocrinol. Metab. 93, 358-362 (2008).

2. Pivonello, R. et al. The treatment of Cushing's disease. Endocr. Rev. 36, 385-486 (2015).

3. Guerin, C. et al. Bilateral adrenalectomy in the $21 \mathrm{st}$ century: when to use it for hypercortisolism? Endocr. Relat. Cancer 23, R131-R142 (2016).

4. Hahner, S. et al. High incidence of adrenal crisis in educated patients with chronic adrenal insufficiency: a prospective study. J. Clin. Endocrinol. Metab. 100 407-416 (2015)

5. Castinetti, F. et al. Long-term results of stereotactic radiosurgery in secretory pituitary adenomas. J. Clin. Endocrinol. Metab. 94, 3400-3407 (2009).

6. Mehta, G. U. et al. Stereotactic radiosurgery for Cushing's disease: results of an international, multicenter study. J. Clin. Endocrinol. Metab. http://dx.doi.org/10.1210/jc.2017-01385 (2017).

7. Burman, P. et al. Radiotherapy, especially at young age, increases the risk for de novo brain tumors in patients treated for pituitary/sellar lesions. J. Clin. Endocrinol. Metab. 102, 1051-1058 (2017)

8. Maione, L. et al. Changes in the management and comorbidities of acromegaly over three decades: the French Acromegaly Registry. Eur. J. Endocrinol. 176 645-655 (2017).

\section{Acknowledgements}

The authors would like to thank the medical staff of the Reference Center for Rare Pituitary Diseases (HYPO), especially H. Dufour and J. Regis.

Competing interests statement

The authors declare no competing interests. 